\title{
Salud y Trabajo Social: un análisis desde las perspectivas de la formación y la investigación
}

\author{
Luis Manuel Rodríguez Otero ${ }^{1}$; $\mathrm{M}^{\mathrm{a}}$ Purificación García Álvarez ${ }^{2}$
}

Recibido: 02/11/2017 / Revisado: 06/02/2018 /Aceptado: 03/07/2018

Disponible on line:

Resumen. La relación entre Trabajo Social y Salud procede de su vinculación inicial con la ciencia médica que potenció por diferentes vías, el Trabajo Social sanitario y el Trabajo Social sociosanitario, quedando patente su inclusión en la esfera social.

La investigación se plantea con una metodología mixta basada en una doble revisión de datos con que se obtiene una triangulación metodológica. La primera consistió en examinar el blog de la Conferencia de Decanos y Directores/as de Trabajo Social de la Universidad española para analizar los planes de estudio de las universidades en las que se oferta el Grado y conocer la representatividad del ámbito de salud. En la segunda se identificaron aquellos artículos de la revista Cuadernos de Trabajo Social que versan sobre salud, para analizar las temáticas y su relación con el contexto. Los resultados revelan que el ámbito de salud tiene una presencia notoria en los planes formativos y en la investigación, siendo las tres temáticas principales: la relación profesional con la salud, las personas mayores con discapacidad y/o dependientes y la salud mental. Asimismo se resaltan diferencias en relación al tipo de centro y el año de publicación. Palabras clave: salud; Trabajo Social; educación; investigación; universidad.

\section{[en] Health and Social Work: an analysis from training and research perspectives}

\begin{abstract}
The relationship between social work and health arises out of its initial links with medical science, which developed healthcare social work and socio-healthcare social work via different routes, making its inclusion as part of the social field clear.

This research was conducted using a mixed methodology based on a double review of data, obtaining methodological triangulation. The first review consisted of examining the blog for the Conference of Spanish University Deans and Heads of Social Work in order to analyse the study plans for the universities at which the undergraduate degree is offered and identify the extent to which health is present. The second identified the articles from the Cuadernos de Trabajo Social journal that addressed health, to permit an analysis of themes and their relationship to the context.

The results show that health has a strong presence in training plans and research. The three main themes are the professional relationship with health, older adults with disabilities and/or dependencies, and mental health. There are also clear differences in relation to the type of centre and year of publication.
\end{abstract}

Key words: health; social work; education; research; university.

Sumario: Introducción. 1. Metodología. 2. Resultados. 3. Discusión. 4. Conclusiones. 5. Referencias bibliográficas.

Cómo citar: Rodríguez Otero, L.M.; García Álvarez, Ma.P. (2019). Salud y Trabajo Social: un análisis desde las perspectivas de la formación y la investigación, en Cuad. trab. soc. 32(1), 123-139.

\footnotetext{
Universidad de Santiago de Compostela, España luismaotero@euts.es

2 Universidad de Santiago de Compostela, España purinaga@euts.es
} 


\section{Introducción}

La salud es un concepto que, dependiendo del momento histórico-social, identifica los elementos que generan el bienestar de las personas; viene a ser una construcción conceptual en la que, en función de los paradigmas científicos en boga en cada momento, inciden en unos aspectos u otros. Actualmente según (Pérez, 2012) los factores tanto personales, como sociales, económicos y ambientales, son los que inciden a nivel individual y poblacional en la salud, interactuando entre sí.

La Organización Mundial de la Salud (OMS) definió el concepto de salud en 1946 como "un estado de completo bienestar físico, mental y social, y no solo la ausencia de molestias o enfermedades". En 1978, fruto de diversas recesiones, se reformuló la definición de salud quedando como: "el perfecto estado de bienestar físico, mental y social y la posibilidad de cualquier persona de aprovechar y desarrollar todas sus capacidades en el orden intelectual, cultural y espiritual", dotándola de un mayor dinamismo, resultando más positiva y real con la situación actual, tal y como plantean Sánchez, Sotomayor, Aquino, Rodríguez, Gil y Gualda (2011, pp. 11-12).

A partir de estas y otras definiciones han surgido planteamientos diferentes que, dependiendo del paradigma con el que se planteen, guían distintos modelos y enfoques (Pérez López, 1990). No obstante, es necesario destacar - como indica Servillo (1999) - que no existe una única Ciencia de la salud, sino que el conocimiento se produce a través de la aportación de distintas ciencias; de este modo podemos diferenciar tres contribuciones: de las ciencias naturales surge la naturalización; de la Ciencia social de la salud surge la socialización y las construcciones discursivas.

En este sentido, Gijón y Colom (2016) ponen un énfasis especial en la repercusión de la definición de la Organización mundial de 1946, al incorporar la dimensión social, lo que facilitó la promoción del modelo bio-psico-social de atención a la salud. Esta perspectiva toma en consideración variables, como el contexto y el movimiento histórico, abogando por lo tanto por la multidisciplonariedad y la interdisciplinariedad, pero también por la relación con la transdisciplinariedad; incluyendo así, las ciencias sociales en el ámbito de la salud y, por lo tanto, al Trabajo Social.
La relación entre el Trabajo Social y la salud proviene de su inicial vinculación a la ciencia médica, que se potencia con la inclusión de la esfera social tanto en la definición de salud como en las legislaciones vinculantes (Pérez López, 1990; Castillo y Abad, 1991; Consejo General del Trabajo Social, s.f.). No debemos olvidar que esta afinidad parte también de la intervención de otras áreas diferentes a la sanitaria, como: servicios sociales, tercer sector o iniciativa privada. En este sentido cabe señalar que, tal y como justifican Gijón y Colom (2016), partiendo de la incorporación de la dimensión social al concepto de salud se identifica una dualidad dimensional de la que derivan posicionamientos teóricos y prácticos compatibles. Encontrando por un lado, la dimensión biológica o naturalista y por el otro, la dimensión social o comprensiva e interpretativa.

Hoy en día la relación entre Trabajo Social y salud queda plasmada en diferentes aportaciones:

1. En el Libro Blanco del Trabajo Social, la sanidad se presenta como uno de los ámbitos profesionales del Trabajo Social con mayor posibilidad de empleo (Núñez, 1999; Vázquez, 2004).

2. En la consideración como un área de formación específica en los títulos universitarios y en los ciclos formativos que dan acceso directo al Grado en Trabajo Social (Consejo General del Trabajo Social, s.f.; Vázquez, 2004).

3. En el reconocimiento del derecho a la salud, la igualdad y la no discriminación, así como en la existencia de determinados grupos de población de mayor riesgo y las acciones de prevención (Ley 14/1986, de 25 de abril, General de Sanidad).

4. En el reconocimiento de los derechos de las personas en situación de dependencia, haciendo especial mención al envejecimiento poblacional y al incremento de personas con alguna enfermedad $u$ otras causas de discapacidad o limitación (Ley 39/2006, de 14 de diciembre, de promoción de la autonomía personal y atención a las personas en situación de dependencia).

Gijón y Colom (2016) ponen señalan con énfasis que el concepto de salud excede los lí- 
mites del ámbito sanitario, siendo fundamental la intervención de las redes adjuntas. En este sentido existen dos conceptos para abordar esta temática:

- Por un lado, tenemos el de Trabajo Social sanitario, que es la especialización que se lleva a cabo en el sistema sanitario (atención primaria, atención sociosanitaria y/o atención especializada), cuya intervención supera la gestión y la coordinación de recursos (Colom, 2010).

- Y por el otro, el Trabajo Social sociosanitario, cuyo objeto radica en la atención sociosanitaria mediante unos recursos existentes, como el apoyo a las intervenciones realizadas desde el ámbito hospitalario y de los centros de salud, y en la red de servicios sociales y servicios sanitarios gestionados de forma coordinada, como señalan los autores.

De esta manera podemos observar que los profesionales del Trabajo Social intervienen en un amplio abanico de situaciones y ante infinidad de necesidades, dependiendo de la conceptualización que utilicen. Por ejemplo, incidiremos en el ámbito de salud, siguiendo la definición de la Organización Mundial de la Salud cuando señala que: "la insuficiencia ponderal, las prácticas sexuales de riesgo, la hipertensión, el consumo de tabaco y alcohol, el agua insalubre, las deficiencias del saneamiento y la falta de higiene", son los principales factores de riesgo en salud (OMS, s.f.). Mientras que, por otro lado, incidiremos en el ámbito sociosanitario si nos guiamos por el Plan Nacional de Acción para la Inclusión Social del Reino de España 2013-2016, al destacar los factores que inciden en la pobreza y la exclusión, como son: 1) el empleo y desempleo; 2) la educación; 3) la vivienda; y 4) la salud; identificando como grupos especialmente vulnerables los compuestos por las personas: sin hogar, con discapacidad, mayores, en situación de dependencia, inmigrantes/migrantes, víctimas de violencia de género y/o discriminación, población gitana, con adicciones y reclusas o ex reclusas (Ministerio de Sanidad, Servicios Sociales e Igualdad, 2014). Razón por la que, tomando en consideración dichos grupos y el ámbito de la salud, cabe destacar los siguientes sectores de población:
- Personas mayores: las características sociodemográficas occidentales describen un perfil social progresivamente envejecido y un aumento de la esperanza de vida; siendo la calidad de vida, el envejecimiento activo y residencial, las necesidades derivadas de los procesos de envejecimiento y los cuidados, elementos de especial interés para el Trabajo Social (Fernández-Riquelme, 2015).

- Personas con discapacidad: las necesidades de las personas con discapacidad son consustanciales, razón por la que se produce la búsqueda de distintas intervenciones dirigidas a cubrir las carencias individuales o sociales. La satisfacción de las necesidades se produce por la familia, como contexto socializador, por el Estado, con la creación de Servicios Sociales y por la actuación de la sociedad civil, con la gestión de recursos sociales (Mercado y García, 2010).

- Personas dependientes: el cuidado continuado de la persona en situación de dependencia dentro de su medio es lo más habitual, lo que provoca la aparición de situaciones que afectan a la salud física y mental de la persona que cuida. Por ello es necesaria la puesta en marcha de recursos de apoyo formal que alivien su labor. Algunos programas instaurados son los psicosociales y psicoeducativos tales como: grupos de apoyo, de ayuda mutua, etc., que fueron llevados a cabo por los trabajadores sociales en coordinación con los Servicios Sociales municipales (Rodríguez, 2013).

- Personas afectadas en su salud mental: la Ley 14/1986, de 25 de abril, General de Sanidad reconoce un título específico, para las personas que se incluyen dentro de este colectivo y reconoce un papel básico del Trabajo Social (art. 20); destacando las funciones de atención, prevención y rehabilitación. La intervención se realiza a través de la red de centros especializados, distribuidos en territorios determinados, ya que como indica Garcés (2010, p. 337), forma parte de la salud integral y se vincula con "las condiciones de vida, con las posibilidades de desarrollo afectivo, emocional, 
intelectual y laboral y con la capacidad de integración al medio social y cultural".

- Personas afectadas por las tóxico-dependencias, violencia, vulnerabilidad y exclusión social: finalmente también cabe destacar que, distintos fenómenos sociales o tipologías de conductas asociadas a problemáticas sociales identificadas como asociales, también son elementos que inciden de forma directa e indirecta en la salud. Así las dependencias de sustancias, la discriminación y los procesos de estigmatización, la violencia, las cuestiones de género y sexualidad y el entorno social de las personas, se identifican como cuestiones de especial interés (Ferro, 2004; Martínez y Ruano, 2013; Lamas, 2014; Arza y Carrón, 2016; Rodríguez-Otero, 2016).

No debemos olvidar que el Trabajo Social sanitario está adscrito al sistema sanitario, llevando a cabo funciones propias del mismo, situándose su integración dentro de los equipos interdisciplinares (Gijón y Colom. 2016). Estos mismos autores, destacan dos grandes necesidades, en relación al Trabajo Social sanitario en el contexto español, como son: por un lado, "delimitar científicamente su objeto de conocimiento y actuación profesional" ( $p$. 17) y, por el otro, de reconocimiento y compromiso.

La importancia de reconocer que la especialización académica y profesional del Trabajo Social Sanitario, además de ser idónea para el desempeño de sus profesionales que se insertan a través del trabajo en equipo en el ámbito sanitario, comporta un compromiso por contribuir a acortar distancias entre la teoría, la investigación y la práctica a la hora de incorporar la dimensión social en salud (idem).

Evidenciada la importancia de la salud en el bienestar de las personas y su vinculación con el Trabajo Social, se presenta a continuación una investigación con los siguientes objetivos.

Objetivo general 1: verificar el grado de implantación de las temáticas de salud en los planes de estudio de Grado en Trabajo Social en las universidades españolas.

Objetivos específicos: a) determinar el número de créditos que se imparten en las uni- versidades españolas de Trabajo Social, con respecto al ámbito de la salud; b) identificar el carácter y la importancia de las asignaturas de salud impartidas en el Grado de Trabajo Social; c) establecer el curso en el que se imparten las asignaturas del ámbito de la salud en las diferentes universidades de la geografía española; d) analizar las temáticas sobre las que versan los contenidos en el ámbito de la salud en el Grado de Trabajo Social; y e) descubrir si el tipo de universidad es determinante en el número de créditos, ámbitos y curso en el que se imparte, tipo de asignatura y la relación existente entre las variables descritas.

Objetivo general 2: analizar las publicaciones referentes al área de salud en la revista Cuadernos de Trabajo Social.

Objetivos específicos: a) descubrir el número de artículos publicados en la revista sobre temáticas de salud, desde 1987 hasta 2017; b) enumerar las temáticas tratadas en los distintos artículos identificados dentro del ámbito de salud; c) identificar la relación entre las temáticas de los artículos publicados y el contexto jurídico-social; y d) comprobar la relación entre las temáticas de los artículos y las asignaturas implantadas en las universidades.

\section{Metodología}

La presente investigación se plantea con una metodología mixta (cualitativa y cuantitativa), basada en la realización de dos revisiones con objeto de obtener una triangulación de los datos y, tal y como indican Hernández, Fernández y Baptista (2003), enriquecer el análisis y agregar complejidad al diseño de estudio, reduciendo las limitaciones de cada método.

La primera, realizada el 13 de julio de 2013, consistió en identificar las universidades españolas que imparten el Grado en Trabajo Social, a partir del blog de la Conferencia de Decanos/as y Directores/as de Trabajo Social de la Universidad Española. Una vez identificadas, se accedió a las 37 universidades en las que se imparte esta carrera (dos de ellas, la de Castilla-La Mancha y la Universidad de Deusto, con dos campus cada una) para revisar los planes de estudios de Grado. El criterio de búsqueda consistió en identificar las materias vinculadas con el ámbito sanitario o afines al mismo. Una vez identificadas, se relacionaron 
con el curso en que se impartía la carga lectiva en créditos ETC y con el tipo de carácter de la materia. Solamente se contabilizaron aquellas materias ofertadas que se impartían realmente.

En septiembre de 2017, se accedió al buscador de revistas online Dialnet, con el fin de identificar aquellas revistas que emergían utilizando la palabra clave "trabajo social". En dicha búsqueda se localizaron 31 revistas, de las cuales 20 tenían su sede editorial en España.

De todas ellas, se seleccionó la revista con mayor impacto e indexación en la Clasificación integrada de revistas científicas (CIRC 2012) y del Sistema de clasificación de revistas científicas de ciencias sociales y humanidades (CARHUS+ 2014): Cuadernos de Trabajo Social de la Universidad Complutense de Madrid. Se accedió a los distintos artículos publicados en el período de 30 años (comprendido entre 1987 y 2017, ambos inclusive) y se identificaron aquellos que hacían mención bien a la salud o bien a algún concepto relacionado con la misma. A continuación, se efectúo un metaanálisis que, como indican Martínez, Sánchez y López (2009): "son revisiones sistemáticas, objetivas y científicas, precisamente porque se desarrollan a través de una serie de etapas claramente establecidas, y que son muy similares a las propias de cualquier investigación empírica" (p. 109). Para ello se utilizó el procedimiento sugerido por dichos autores, que consiste en: 1) formular el problema; 2) realizar la búsqueda de la literatura; 3) codificar los estudios; 4) realizar un análisis estadístico e interpretar los datos; y 5) publicar el metaanálisis.

Para la codificación de los estudios se originó un cuadro en la que se identificó: autor/ es, año de publicación, temática del artículo, número de artículos que incluía la revista y número de artículos de salud incluidos en la publicación. Conseguida la información, se realizó un análisis relacionando la terminología utilizada en los títulos de los artículos, con los nombres de las materias implantadas en los estudios de Trabajo Social. Este análisis consistió en un proceso de categorización emergente de las temáticas (Hernández, Fernández y Baptista, 2003). Así mismo, respecto a los artículos, se identificaron las palabras más frecuentes, extrayendo una tabla de frecuencias y una nube de palabras. En dicho proceso, se utilizaron como criterios de análisis la frecuencia de las 100 palabras más utilizados con 5 letras al menos, en lo que se incluyeron sinónimos y derivadas. De esta manera, para la nube de palabras no se utilizaron criterios de restricción. A partir de dichas palabras se realizó un proceso de categorización emergente. Como herramientas de análisis se utilizaron los programas informáticos SPSSv.25 y el NVivo11.

\section{Resultados}

Analizadas todas las universidades de la geografía española (37 en total), en las que se cursan los estudios de Trabajo Social, en el curso académico 2016/2017, vimos que en 23 de ellas se imparte una única materia en todo el currículo con el título de salud y con una carga académica de 6 créditos ECTS. Es de destacar que existen varias universidades, como la Universidad Complutense de Madrid, la universidad Pablo Olavide de Sevilla, la Universidad de Santiago de Compostela y la Universidad de Valladolid en las que esta materia se imparte en dos asignaturas (con un peso de 6 créditos ECTS cada una de ellas); también en la Universidad de La Rioja y la Universidad de Valencia esta materia se organiza en dos asignaturas, pero con una carga de 4,5 créditos ECTS cada una de ellas.

Gráfico 1: Carga docente de las asignaturas de salud impartidas en las universidades

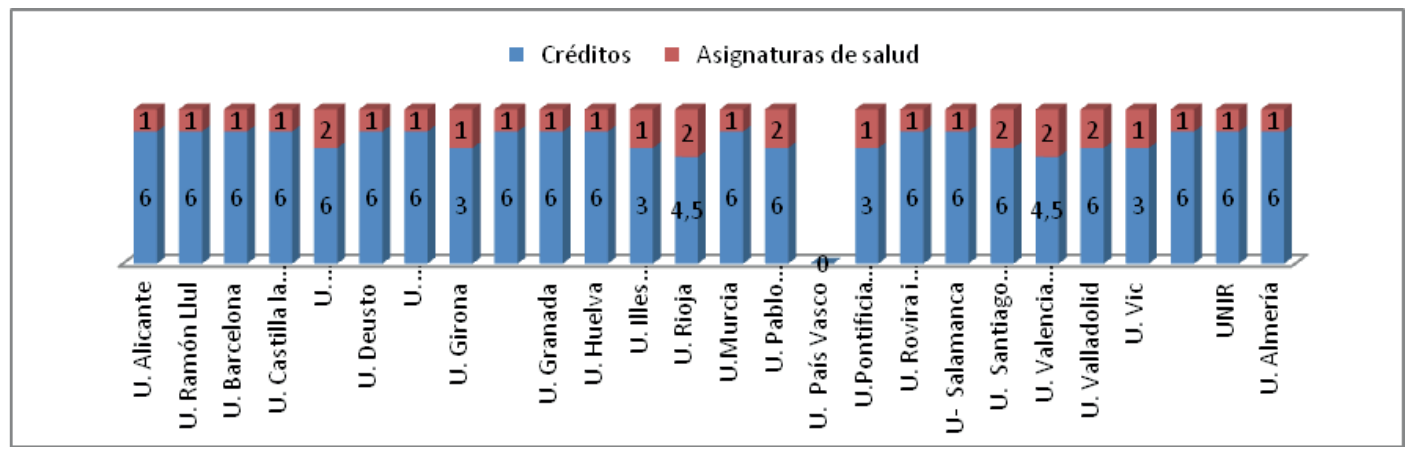

Fuente: Elaboración propia. 
Solo cuatro universidades tienen menor representación curricular en el ámbito de salud, al haber implantado una sola asignatura de 3 créditos ECTS (la Universitat de Girona, la Universidad de Comillas, la Universidad de les
Illes Balears y la Universitat de Vic). Es necesario constatar, que no tenemos conocimiento de las asignaturas impartidas en la Universidad del País Vasco.

Gráfico 2: Peso de las asignaturas de salud en los planes de estudio

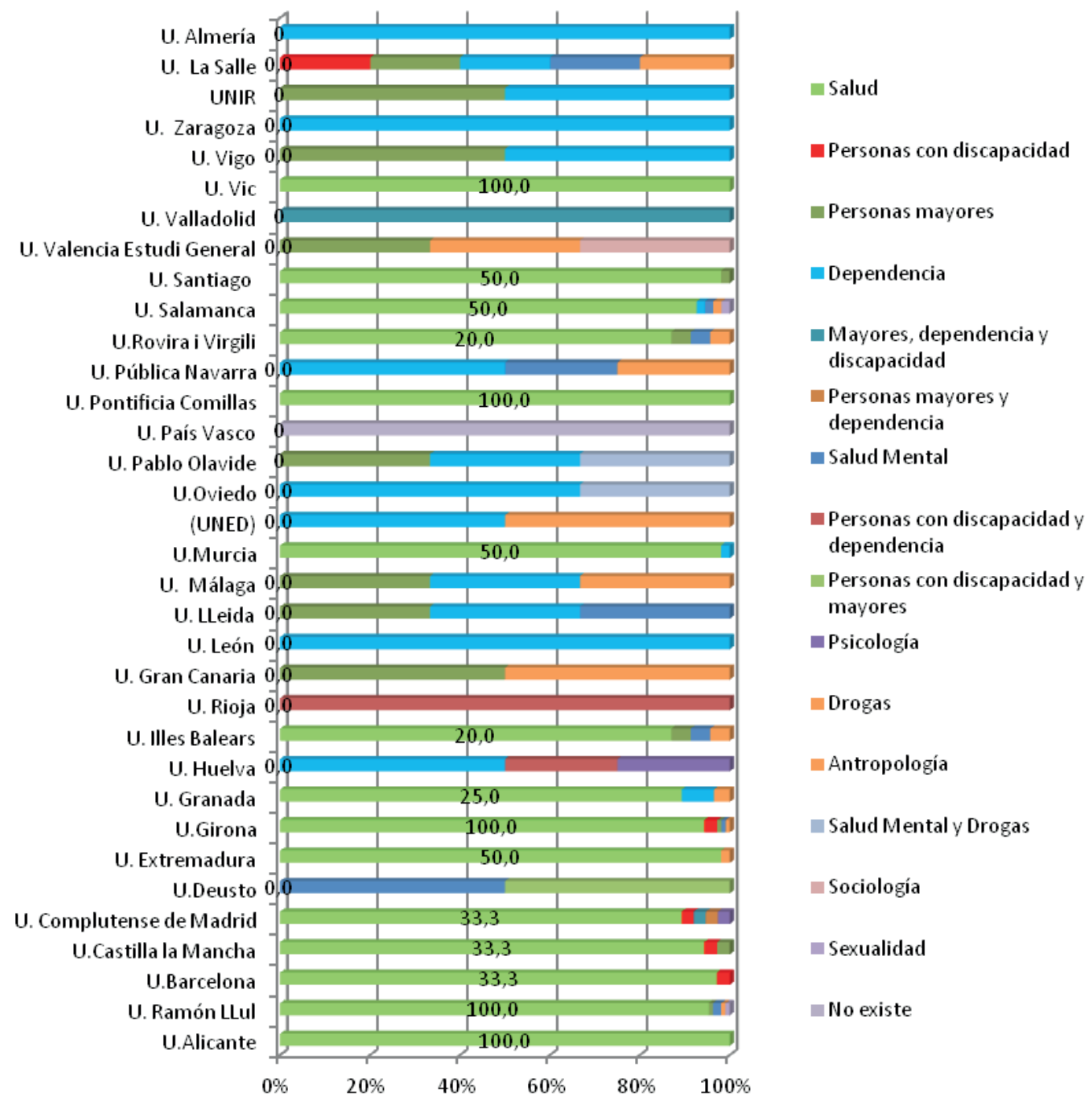

Fuente: Elaboración propia.

Como se puede apreciar, el área de salud es el que tiene mayor representatividad, tanto es así que algunas de las universidades, como la Universidad de Alicante o la Universidad de Comillas (implantadas en una sola asignatura), abarcan el 100 por cien de los créditos ECTS del propio ámbito de la salud. Sin embargo otras imparten la mitad o una tercera parte de los créditos en el ámbito de la salud, completando hasta el número total de crédi- tos, con los cursados en otros ámbitos como: el de dependencia, el de las personas con discapacidad o el de las personas mayores. No obstante, se observa que en otras universidades no se cursan asignaturas específicas del ámbito de la salud, aunque sí se imparten en asignaturas relacionadas con diferentes sectores de población.

Otro punto importante indagado es el del/ los curso/os en los que están establecidas las 
asignaturas de esta temática. Así vemos que en 12 universidades se imparte una sola asignatura en el $3^{\circ}$ curso, en otras cinco se imparten en $4^{\mathrm{o}} \mathrm{y}$ en tres universidades más (la Universidad de La Rioja, la Universidad Complutense y la Universidad de Barcelona), se cursa en $3^{\circ}$ y en $4^{\circ}$. Se destaca que, solo en 5 universidades esta materia se oferta en el $2^{\circ}$ curso.

Figura 1: Cursos en los que se imparten las asignaturas del ámbito de la salud

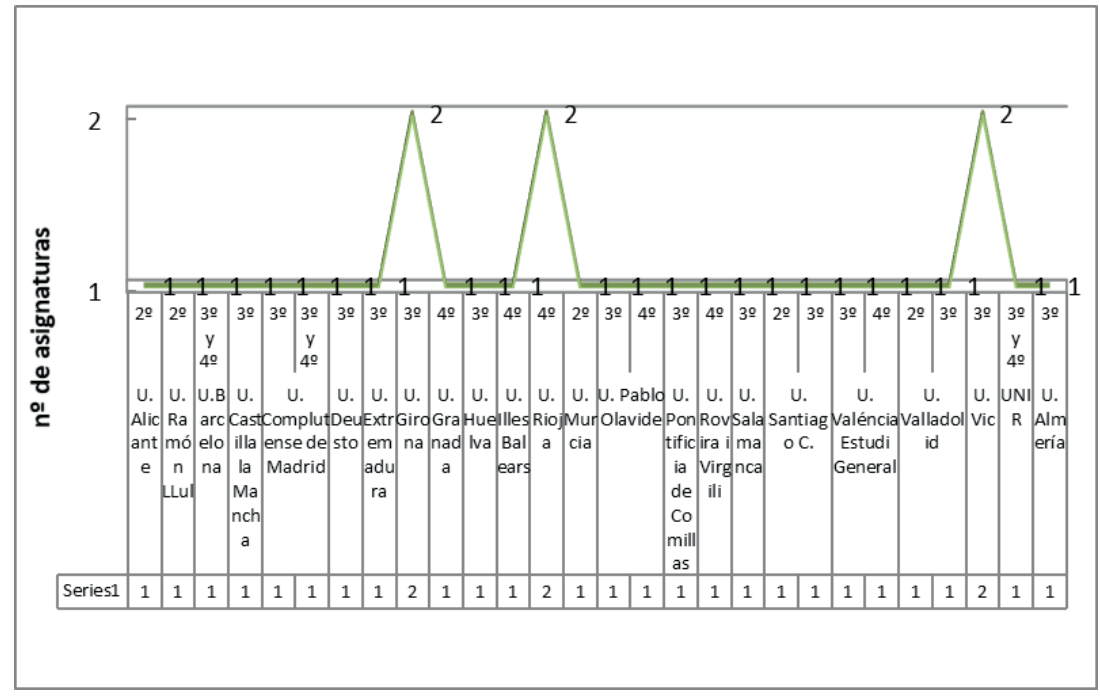

Fuente: Elaboración propia.

A partir de los planes de estudio de Grado de Trabajo Social, comprobamos que de las 127 asignaturas del área de la salud, el peso se reparte de la siguiente forma: el propio ámbito de salud lo representan 31 asignaturas $(24,4$ por ciento); le sigue el ámbito de la dependencia con 26 asignaturas (20,5 por ciento), posteriormente el ámbito de personas con discapacidad con 16 asignaturas $(12,6$ por ciento) $y$, en cuarto lugar y sin mucha diferencia encontramos el ámbito de las personas mayores en 15 asignaturas (11,8 por ciento). El resto de las materias instauradas tienen una menor representatividad y comparten varios conocimientos tales como: personas mayores y dependencia o personas con discapacidad y mayores.

Como se observa en el anexo II (ver figura 9) y tomando en consideración todas las asignaturas distribuidas en los planes de estudio dentro el ámbito de salud, se evidencia que hay materias que tienen contenidos análogos, aunque presentan un número de créditos diferente y se adjudican a distintos cursos y hasta con enfoques diferenciados.

Gráfico 3: Análisis de las asignaturas según los créditos, cursos y tipo de universidad

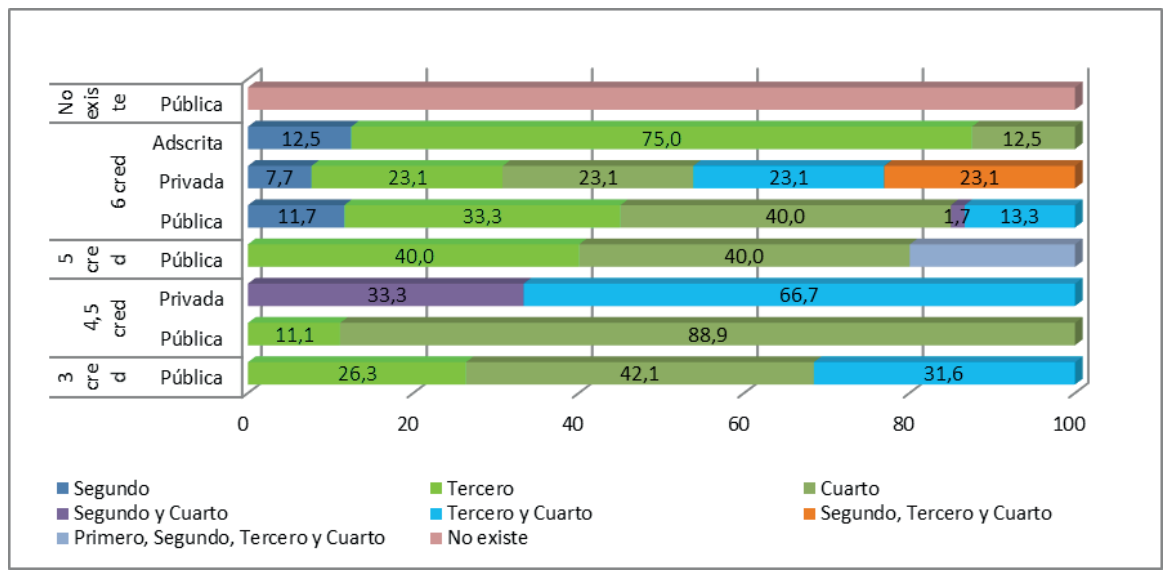

Fuente: Elaboración propia. 
Es relevante destacar que, según la tipología de la universidad que se trate, hay grandes divergencias, así vemos que:

- En las universidades públicas, además de claras diferencias en las materias de salud, también se visualizan discrepancias en lo referente al número de créditos y al curso en el que están asignadas; tanto es así que aquellas que tienen 6 créditos ECTS se ofertan en $4^{\circ}$ (en el 40 por ciento de los casos) y en $3^{\circ}$ ( en el 33,3 por ciento), mientras que las asignaturas de 4,5 créditos ECTS se cursan en $4^{\circ}$ ( 88,9 por ciento), con un total de 3 asignaturas tanto obligatorias como optativas;

- En las universidades adscritas, todas las asignaturas implantadas -obligatorias u optativas - tienen 6 créditos ECTS ( 2 de cada tipo), además el 75 por ciento de esos créditos se cursan en $3^{\circ}$, quedando distribuido el otro 25 por ciento, a partes iguales, entre $2^{\circ}$ y $4^{\circ}$ cursos; y

- Las universidades privadas cuentan con materias de 6 y de 4,5 créditos ECTS en di- ferentes cursos, aunque representan el 66,7 por ciento en aquellas de 6 créditos ECTS implantados en $3^{\circ}$ y en $4^{\circ}$, siendo más elevada la oferta de asignaturas optativas que de obligatorias.

No debemos olvidar que si relacionamos el tipo de materia con el ámbito docente (tipo de Universidad) vemos que mientras en las universidades públicas y privadas hay un mayor abanico de materias optativas, en las universidades adscritas el peso de las materias está muy igualado (entre optativas y obligatorias).

Analizando los artículos publicados en la revista citada sobre temáticas de salud desde 1987 hasta 2017, se observa que de los 623 artículos publicados (en los 30 volúmenes y 37 números), 87 versan sobre temáticas de salud, lo cual representa el 13,8 por ciento. Los volúmenes que recogen un mayor número de artículos y en los que la representatividad es mayor se corresponden con los publicados en 1995 (66,7 por ciento), 2001 (54,6 por ciento) y 2010 (52,2 por ciento).

Tabla 1: Artículos publicados en la revista Cuadernos de Trabajo Social

\begin{tabular}{|c|c|c|c|c|c|c|c|c|c|}
\hline Año & $\begin{array}{l}\text { Volumen } \\
\text { y número }\end{array}$ & $\begin{array}{l}\text { Artículos } \\
\text { publicados }\end{array}$ & $\begin{array}{l}\text { Artículos } \\
\text { Salud }\end{array}$ & $\begin{array}{l}\% \text { artículos } \\
\text { salud }\end{array}$ & Año & $\begin{array}{l}\text { V o l u - } \\
\text { men } \\
\text { y núme- } \\
\text { ro }\end{array}$ & $\begin{array}{l}\text { Artículos } \\
\text { publicados }\end{array}$ & $\begin{array}{l}\text { Artículos } \\
\text { Salud }\end{array}$ & $\begin{array}{l}\% \text { artículos } \\
\text { salud }\end{array}$ \\
\hline \multirow[t]{2}{*}{2017} & $30(1)$ & 16 & 1 & 6,25 & 2005 & 18 & 20 & 3 & 15,00 \\
\hline & $30(2)$ & 15 & 3 & 20,00 & 2004 & 17 & 16 & 5 & 31,25 \\
\hline \multirow[t]{2}{*}{2016} & $29(1)$ & 10 & 1 & 10,00 & 2003 & 16 & 18 & 1 & 5,56 \\
\hline & $29(2)$ & 16 & 0 & 0,00 & 2002 & 15 & 19 & 1 & 5,26 \\
\hline \multirow[t]{2}{*}{2015} & $28(1)$ & 11 & 0 & 0,00 & 2001 & 14 & 22 & 12 & 54,55 \\
\hline & $28(2)$ & 10 & 0 & 0,00 & 2000 & 13 & 20 & 4 & 20,00 \\
\hline \multirow[t]{2}{*}{2014} & $27(1)$ & 16 & 1 & 6,25 & 1999 & 12 & 18 & 3 & 16,67 \\
\hline & $27(2)$ & 20 & 0 & 0,00 & 1998 & 11 & 19 & 4 & 21,05 \\
\hline \multirow[t]{2}{*}{2013} & $26(1)$ & 22 & 1 & 4,55 & 1997 & 10 & 17 & 1 & 5,88 \\
\hline & $26(2)$ & 18 & 3 & 16,67 & 1996 & 9 & 19 & 1 & 5,26 \\
\hline \multirow[t]{2}{*}{2012} & $25(1)$ & 18 & 2 & 11,11 & 1995 & 8 & 18 & 12 & 66,67 \\
\hline & $25(2)$ & 19 & 0 & 0,00 & 1994 & 7 & 23 & 2 & 8,70 \\
\hline 2011 & 24 & 12 & 0 & 0,00 & 1993 & 6 & 15 & 1 & 6,67 \\
\hline 2010 & 23 & 23 & 12 & 52,17 & 1992- 1991 & $4-5$ & 28 & 3 & 10,71 \\
\hline 2009 & 22 & 17 & 6 & 35,29 & 1990 & 3 & 11 & 1 & 9,09 \\
\hline 2008 & 21 & 19 & 0 & 0,00 & 1989 & 2 & 16 & 1 & 6,25 \\
\hline 2007 & 20 & 17 & 0 & 0,00 & 1988 & 1 & 14 & 0 & 0,00 \\
\hline 2006 & 19 & 19 & 0 & 0,00 & 1987 & 0 & 12 & 1 & 8,33 \\
\hline
\end{tabular}

Fuente: elaboración propia 
Desataca también que el incremento en el número de artículos sobre salud es irregular, identificándose periodos en los que a éste le sigue un descenso agudo (1995-1997, 20012003 y 2010-2011). Así mismo, se constata que no existe relación entre el incremento o la disminución del número de artículos en la revista con el número de artículos sobre salud.

Respecto a las temáticas tratadas en los distintos artículos identificados dentro del ámbito de salud, se observa que existe una pluralidad de aspectos. Así, tras realizar el conteo y análisis de la frecuencia de palabras incluidas en los títulos de los artículos, se aprecia que la temática central y la que ocupa un espacio destacado es la cuestión social (39 palabras). No obstante, también destacan las frecuencias de otras palabras tales como: trabajo (19 palabras), personas (16), mental (15), salud (13), mayores (11), atención (10) y drogodependencias (9).

Figura 2: Nube de palabras títulos artículos.

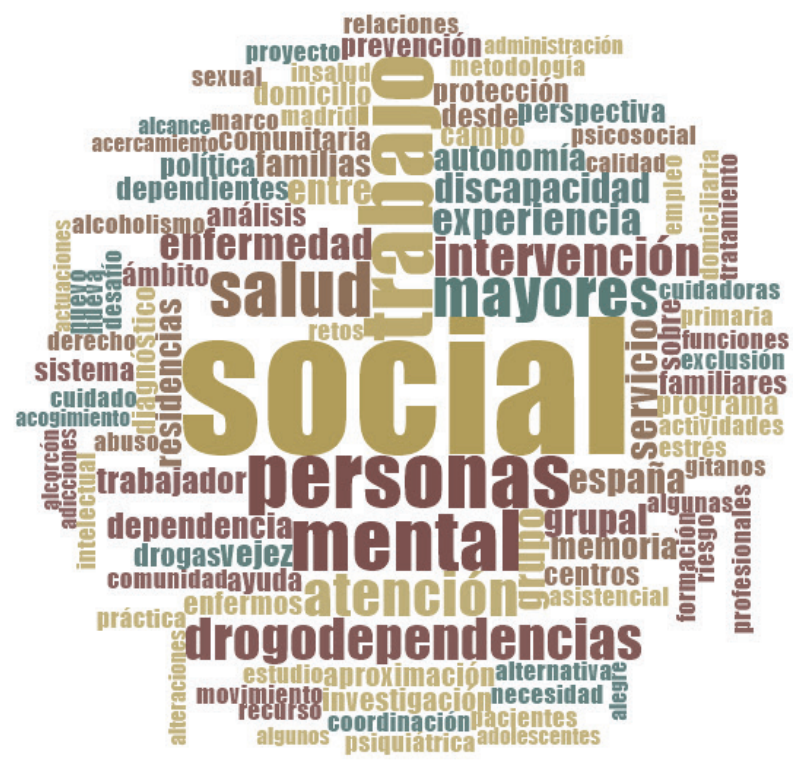

Fuente: Elaboración propia.

Se identifican otras palabras que, aunque de menor frecuencia, son también representativas; entre ellas destacan: discapacidad, enfermedad, experiencia, autonomía, servicios, residencias, memoria, grupal, dependencia y trabajador. En este sentido cabe resaltar, tanto en la formación académica como en los artículos identificados, que existe una tendencia a incluir temáticas vinculadas a la salud pública, el Trabajo Social clínico y, en menor medida, el Trabajo Social sociosanitario, que registra una mayor presencia a partir de la aparición de la Ley de promoción de la autonomía personal y atención a las personas en situación de dependencia.

Por otro lado, tras realizar el proceso de categorización emergente sobre las temáticas de los artículos, tal y como se observa en la siguiente tabla, existe una pluralidad de términos. 
Tabla 2: Categorías emergentes de las temáticas en artículos

\begin{tabular}{|l|l|l|}
\hline Temática & $\mathrm{N}^{\mathrm{o}}$ & $\%$ \\
\hline Salud & 9 & 10,34 \\
\hline Salud mental & 18 & 20,68 \\
\hline Personas con discapacidad & 9 & 10,34 \\
\hline Sexualidad & 4 & 4,59 \\
\hline Dependencia & 8 & 9,19 \\
\hline Personas mayores & 16 & 17,24 \\
\hline Drogas & 18 & 20,68 \\
\hline Psicología, antropología, sociología y Salud & 1 & 1,14 \\
\hline Atención domiciliaria & 5 & 5,74 \\
\hline Atención residencial & 1 & 1,14 \\
\hline Servicios Sociales comunitarios & 1 & 1,14 \\
\hline Cuidadores & 1 & 1,14 \\
\hline Voluntariado & 1 & 1,14 \\
\hline Objetores de conciencia & 1 & 1,14 \\
\hline Familia & 1 & 1,14 \\
\hline Trabajo Social de grupos & 4 & 4,59 \\
\hline Trabajo Social con comunidad & 1 & 1,14 \\
\hline Redes sociales & 2 & 2,28 \\
\hline Intervención en crisis & 1 & 1,14 \\
\hline Menores & 1 & 1,14 \\
\hline Violencia & 2 & \\
\hline Género & 1 & 1,14 \\
\hline Síndrome de bournout & 3 & 3,44 \\
\hline Política social & 1 & 1,14 \\
\hline Ética & 1 & 1,14 \\
\hline Vulnerabilidad/ exclusión & 2,28 \\
\hline
\end{tabular}

Fuente: Elaboración propia

Dentro del conjunto de categorías se aprecia que en 23 artículos la temática es mixta, siendo 20 dobles y 3 triples. Por otro lado, se identifica que las principales temáticas son salud mental y drogas $(20,7$ por ciento respectivamente), aunque las temáticas de dependencia, personas con discapacidad, personas mayores y servicios-recursos vin- culados a estas temáticas representan el 44,8 por ciento.

Analizando los periodos de tiempo en los que sobresalen las publicaciones de artículos sobre salud y las temáticas sobre las que versan, comprobamos que tienen una mayor presencia en los años 1995, 2001, 2004, 20092010, 2012-2013 у 2016-2017.

Gráfico 4: Artículos publicados según año y temática

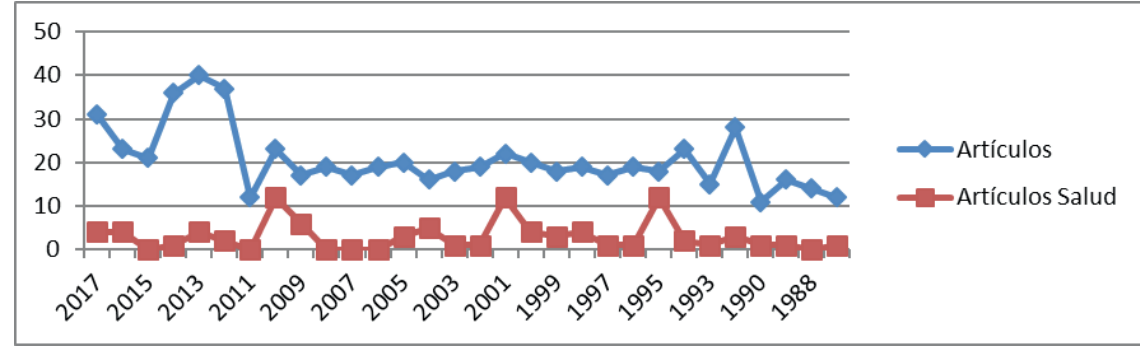

Fuente: Elaboración propia. 
Tomando en consideración los periodos señalados y los contenidos identificados se observa que, en 1995, la temática más frecuente era drogas; en 2001, personas mayores; en
2004, existe una pluralidad de temáticas; entre 2009 y 2010 salud mental; entre 2012-2013 dependencia y discapacidad y entre 2016-2017 diferentes temáticas.

Cuadro 1: Temáticas de los artículos según periodos destacados

\begin{tabular}{|c|c|c|c|c|c|c|}
\hline Temática & 1995 & 2001 & 2004 & $2009-2010$ & $2012-2013$ & 2016-2017 \\
\hline Salud & $\mathrm{x}$ & & & & & \\
\hline Salud mental & & $\mathrm{x}$ & $\mathrm{x}$ & $\mathrm{X}$ & $\mathrm{x}$ & $\mathrm{x}$ \\
\hline Personas con discapacidad & & & & $\mathrm{X}$ & $\mathrm{x}$ & $\mathrm{x}$ \\
\hline Sexualidad & & & & $\mathrm{X}$ & $\mathrm{x}$ & \\
\hline Dependencia & & $\mathrm{x}$ & $\mathrm{x}$ & $\mathrm{X}$ & $\mathrm{x}$ & $\mathrm{x}$ \\
\hline Personas mayores & & $\mathrm{x}$ & & $X$ & & \\
\hline Drogas & $\mathrm{x}$ & & $\mathrm{x}$ & $\mathrm{X}$ & $\mathrm{x}$ & $\mathrm{x}$ \\
\hline Psicología, antropología, sociología y Salud & & & $\mathrm{x}$ & & & \\
\hline Atención domiciliaria & & $\mathrm{x}$ & & $\mathrm{X}$ & & \\
\hline \multicolumn{7}{|l|}{ Atención residencial } \\
\hline \multicolumn{7}{|l|}{ Servicios Sociales comunitarios } \\
\hline Cuidadores & & $\mathrm{x}$ & & & & \\
\hline Voluntariado & & & $\mathrm{x}$ & & & \\
\hline Objetores de conciencia & $\mathrm{x}$ & & & & & \\
\hline \multicolumn{7}{|l|}{ Familia } \\
\hline Trabajo Social de grupos & $\mathrm{x}$ & & & & & \\
\hline Trabajo Social con comunidad & & & & $\mathrm{X}$ & & \\
\hline \multicolumn{7}{|l|}{ Redes sociales } \\
\hline Intervención en crisis & & & & $\mathrm{X}$ & & \\
\hline \multicolumn{7}{|l|}{ Menores } \\
\hline Violencia & & & & & $\mathrm{x}$ & \\
\hline \multicolumn{7}{|l|}{ Género } \\
\hline Síndrome de bournout & & $\mathrm{x}$ & $\mathrm{x}$ & & & \\
\hline Política social & $\mathrm{x}$ & & & & & \\
\hline \multicolumn{7}{|l|}{ Ética } \\
\hline Vulnerabilidad/ exclusión & & & & & & $\mathrm{x}$ \\
\hline
\end{tabular}

Fuente: Elaboración propia.

Observamos que temáticas, como salud mental, dependencia y drogas, constituyen una constante en la mayor parte de los periodos analizados, y que en el periodo 2009-2010 se registra un mayor número de temáticas abordadas.

Cuando se toma en consideración el contexto social de los periodos identificados, la publicación de legislaciones sobre temáticas de política social en España, el contexto político-ideológico y los acontecimientos históricos relevantes, así como las temáticas publicadas en la revista, se observa que:

- Durante los años en los que las ideologías de izquierdas adquirían el poder, se produ- 
cía un mayor número de derechos sociales y políticas sociales (ver Anexo I).

- Se identifica que las temáticas de los artículos están estrechamente relacionadas con el contexto social. Así, debido al aumento de población drogodependiente durante la década de 1980 y el incremento de servicios específicos e intervenciones profesionales, tiene como apogeo a nivel científico el año 1995. Por otro lado, debido a la promulgación de leyes - como la ley de la Seguridad Social (1994) y de reconocimiento del grado de discapacidad (1999) - junto con el envejecimiento poblacional en España, se incrementa el interés de la comunidad científica por temáticas vinculadas a las personas mayores y/o a la discapacidad. Con la modificación del sistema de atención de personas con problemas vinculados a la salud mental, emerge el interés por dar respuesta a este colectivo, siendo en los años 2009 y 2010 cuando se evidencia un mayor número de publicaciones. Por otro lado, tras la publicación de la Ley de Dependencia (2006) y los efectos de la crisis económica en España, a partir de 2008, emerge cierta preocupación gremial por las sucesivas modificaciones a la ley inicial, y de este modo en los años 2012 y 2013 las temáticas más comunes fueron dependencia y discapacidad. Finalmente se identifican temáticas similares en 2016 y 2017, además de aspectos derivados de la vulnerabilidad y la exclusión social, siendo este periodo el punto álgido en la políticas de austeridad (ver Anexo I).

En relación a las temáticas de los artículos y las asignaturas implantadas en las universidades destaca que las temáticas de las asignaturas vinculadas al ámbito de salud son 8 (salud, salud mental, personas con discapacidad, sexualidad, dependencia, personas mayores, drogas y psicología-antropología-sociología y salud), así como la combinación de distintas temáticas.

Cuadro 2: Temáticas de los artículos identificados

\begin{tabular}{|c|c|c|}
\hline \multicolumn{2}{|l|}{ Temáticas } & \multirow{2}{*}{$\begin{array}{l}\mathrm{N}^{\mathrm{o}} \\
82\end{array}$} \\
\hline \multirow[t]{8}{*}{ Similar a asignaturas } & Salud & \\
\hline & Salud mental & \\
\hline & Personas con discapacidad & \\
\hline & Sexualidad & \\
\hline & Dependencia & \\
\hline & Personas mayores & \\
\hline & \begin{tabular}{|l|} 
Drogas \\
\end{tabular} & \\
\hline & Psicología, antropología, sociología y Salud & \\
\hline \multirow[t]{6}{*}{ Servicios } & Atención domiciliaria & \multirow[t]{6}{*}{10} \\
\hline & Atención residencial & \\
\hline & Servicios Sociales comunitarios & \\
\hline & Cuidadores & \\
\hline & Voluntariado & \\
\hline & Objetores de conciencia & \\
\hline \multirow{5}{*}{$\begin{array}{l}\text { Niveles de intervención y modelos de inter- } \\
\text { vención }\end{array}$} & Familia & \multirow[t]{5}{*}{9} \\
\hline & Trabajo Social de grupos & \\
\hline & Trabajo Social con comunidad & \\
\hline & Redes sociales & \\
\hline & Intervención en crisis & \\
\hline \multirow[t]{7}{*}{ Otros aspectos } & Menores & \multirow[t]{7}{*}{11} \\
\hline & Violencia & \\
\hline & Género & \\
\hline & Síndrome de bournout & \\
\hline & Política social & \\
\hline & Ética & \\
\hline & Vulnerabilidad/ exclusión & \\
\hline
\end{tabular}

Fuente: Elaboración propia 
Igualmente, en los artículos de Cuadernos de Trabajo Social se identifican, por un lado temáticas similares a las asignaturas impartidas en los grados de Trabajo Social $(71,1$ por ciento del total de artículos) y, por el otro, los elementos relacionados con servicios $(11,3$ por ciento), niveles y modelos de intervención ( 7,9 por ciento) o con otras temáticas $(9,7$ por ciento). Así mismo, se evidencian ítems categorizados dentro de la taxonomía de servicios vinculados a la intervención con personas mayores, con discapacidad y/o dependientes (Atención domiciliaria, Atención residencial y Cuidadores), siendo el 77 por ciento la representación de estos artículos, sumados a los anteriores.

Finalmente cabe destacar que la temática más frecuente en las asignaturas impartidas en las universidades es la de "salud" y su vinculación o relación con el Trabajo Social, mientras que en los artículos es la de "intervención con personas mayores, con discapacidad y/o dependientes" ( 31,5 por ciento), seguido de "salud mental" ( 16,3 por ciento) y "drogas" $(15,5$ por ciento).

\section{Discusión}

En la presente investigación se observa que todas la universidades españolas en las que se imparte el Grado en Trabajo Social, a excepción de una, incluyen asignaturas del área de salud en su planes académicos, lo que evidencia, tal y como señalan Pérez López (1990), Castillo y Abad (1991) y el Consejo General del Trabajo Social (s.f.), que la vinculación entre el Trabajo Social y la salud es indiscutible y evidenciable en las prácticas profesionales; en su formación académica, empleabilidad y legislaciones vinculantes (Núñez, 1999; Vázquez, 2004; . No obstante, se observa que, dependiendo de la sede, la carga académica asignada a esta área varía notoriamente; lo cual, también pone de manifiesto, que no existe un consenso respecto a su influencia, siendo otras áreas o ciencias afines al Trabajo Social las que tienen mayor peso (Vázquez, 2004). Así mismo, se identifica una mayor presencia de connotaciones del Trabajo Social sanitario que del Trabajo Social sociosanitario, aunque la sede de la Universidad resulta determinante y su peso académico es muy bajo. Igualmente, queda constatada una situación semejante con respecto a las publicaciones científicas. Así, en la revista Cuadernos de Trabajo Social la cobertura de los artículos sobre temáticas de salud representan el 13,8 por ciento del total, lo cual afianza su hegemonía o prevalencia dentro de la disciplina y como un área específica de la profesión (Consejo General del Trabajo Social, s.f.). A este respecto cabe destacar la reflexión que realizan Gijón y Colom (2016), quienes resaltan la complejidad existente en el contexto español al respecto. Puesto que no existe una delimitación científica del objeto de conocimiento y acción profesional, así como un reconocimiento a la especialización del Trabajo Social sanitario; siendo la confusión entre ambas tipologías fuertemente arraigada.

En referencia a las temáticas destacadas tanto en las asignaturas como en las publicaciones se observa que, al igual que indican la Organización Mundial de la Salud (OMS, s.f.) y el Ministerio de Sanidad, Servicios Sociales e Igualdad (2014), existe una pluralidad de factores interrelacionados. Se evidencian áreas en ambos elementos objeto de estudio (asignaturas y artículos) relativos a: 1) la relación de la profesión y la salud -como Gil (2010) o Brufao (1994)- tal y como señalan anteriormente otros autores (Vázquez, 2004; Consejo General del Trabajo Social, s.f.); 2) la Salud mental de las personas y del entorno de quienes tienen necesidades derivadas, tal y como se observa en artículos, como los de Deviat (2017) o Ureña (2010); 3) vinculantes a personas con algún tipo de discapacidad (Casal, 2010; del Álamo y Ruíz, 2017); 4) personas mayores (Puente, 2001, Cury, 2009); 5) personas dependientes o que están vinculadas a éstas (Cordero y Palacios, 2017); 6) toxicodependencias (Arza y Carrón, 2016); y 7) cuestiones relacionadas con el género y la sexualidad (Sanz, 2009; Martínez y Ruano, 2013). En los artículos científicos se constata que, por un lado, las temáticas están vinculadas en cierta forma al contexto sociohistórico (realidad social) y las necesidades del momento; por otro lado, la existencia de otras temáticas, como la presentación, teorización o descripción de servicios o recursos vinculantes (Núñez y Cerón, 2001; Robles y Lara, 2009); los niveles y modelos de intervención (Millán, 2005; Colama, 2009) y otras áreas también identificadas por la Organización Mundial de la Salud (OMS, s.f.) y el Ministerio de Sanidad, Servicios Sociales e Igualdad (2014), como son: los menores, la violencia, el género, el síndrome de bournout, la política social, la ética o la vulnerabilidad 
y la exclusión (Millán, 2005; Martínez y Ruano, 2013; Deviat, 2017). Siendo las temáticas vinculadas al Trabajo Social clínico y la salud pública las más frecuentes en las publicaciones identificadas, destacando que en los últimos años, se ha incrementado el interés por temáticas relativas al Trabajo Social sociosanitario, tras la publicación de legislaciones vinculantes a los colectivos anteriormente señalados (Ley de Dependencia, Rentas Básicas, Discapacidad, etc.).

\section{Conclusiones}

Examinados los planes de estudio de todas las universidades españolas en las que se imparten los estudios de Grado de Trabajo Social, comprobamos que en 25 de ellas se ofertan asignaturas del ámbito de salud, implantadas en 127 asignaturas. Dentro de la temática de la salud, el mayor peso recae en el propio ámbito, repartido en 31 asignaturas (24,4 por ciento), seguido del ámbito de la dependencia, representado por 26 materias $(20,5$ por ciento) y en tercer lugar, se encuentra el ámbito de personas con discapacidad que se distribuyen en 16 asignaturas (12,6 por ciento). La mayoría de ellas son de 6 créditos ECTS y se distribuyen en una sola asignatura, entre $3^{\circ}$ y $4^{\circ}$ curso, aunque encontramos algunas universidades que ofertan dos materias con el mismo peso ( 6 créditos ECTS) y otras, en las que esta docencia tiene menor peso (4,5 créditos ECTS).

Solo tres Universidades, cubren el 100 por cien de los créditos destinados a este ámbito con las asignaturas de la salud, mientras que la gran mayoría completan esta carga lectiva impartiendo otras asignaturas relacionadas con la salud como: dependencia, personas con discapacidad o personas mayores.

Se observa que el tipo de Universidad es determinante en relación al número de créditos, ámbitos y cursos en los que se imparten las asignaturas del ámbito de la salud, tanto es así que: en las universidades adscritas todas las asignaturas tienen un peso de 6 créditos ECTS, se dividen a partes iguales entre materias obligatorias y optativas y en el 75 por ciento de los casos se imparten en el $3^{\circ}$ curso. En las universidades públicas varía el porcentaje de créditos y cursos, pues las materias de 6 créditos ECTS (mayoritariamente optativas) se cursan en el $4^{\circ}$ (40 por ciento de los casos) y en el $3^{\circ}$ curso (33,3 por ciento). Mientras que en las universidades privadas el porcentaje de asignaturas implantadas es más bajo $(23,1$ por ciento) tanto en el $3^{\circ}$ como en el $4^{\circ}$ curso y con la misma carga en la docencia obligatoria que en la optativa.

Analizando los artículos publicados en Cuadernos de Trabajo Social sobre las temáticas de la salud, se observa que esta temática representa el 13,8 por ciento de los artículos y que los años con mayor número de publicaciones son 1995 (66,7 por ciento), 2001 (54,5 por ciento) y 2010 (52,2 por ciento), siendo las temáticas más frecuentes drogas, mayores y salud mental respectivamente en cada año.

En las temáticas tratadas en los títulos de los artículos dentro del ámbito de la salud, tras realizar el análisis de la frecuencia de palabras incluidas, se observa que la que ocupa un espacio destacado, es la cuestión social (con 39 repeticiones). No obstante, también destacan las frecuencias de otras palabras tales como: trabajo, personas, mental, salud, mayores, etc.

A través del proceso de categorización emergente sobre las temáticas de los artículos, vemos que existe una pluralidad de términos. Así, en 23 artículos la temática es mixta, siendo en 20 dobles y en 3 triple. Las temáticas que han tenido una constante en las publicaciones son: salud mental, dependencia y drogas.

Como datos relevantes establecemos que: las temáticas de las asignaturas vinculadas al ámbito de la salud son 8 (salud, salud mental, personas con discapacidad, sexualidad, dependencia, personas mayores, drogas y disciplinas afines). Que los artículos se identifican por un lado con las asignaturas impartidas en los grados de Trabajo Social (71 por ciento), con los servicios (11 por ciento), con niveles y modelos de intervención ( 7,95 por ciento) y van disminuyendo sucesivamente.

Considerando el contexto social de los periodos identificados vemos que, con gobiernos de ideologías progresistas, se generan más abundancia de derechos y políticas sociales y que las temáticas de los artículos están estrechamente ligados con el contexto social como ocurre con la drogadicción en los años ochenta o el incremento de servicios específicos e intervenciones profesionales en la década de los noventa.

Finalmente se constata que existe una relación directa entre las temáticas de las asignaturas impartidas y los artículos científicos sobre salud identificados. Siendo en estos últimos donde se da una mayor cobertura, ofreciendo 
una perspectiva más amplia del campo de la salud en la profesión. Es por ello por lo que se considera necesario tomar en consideración los elementos expuestos y promover un espacio de reflexión y debate, dirigido a incluir en la formación de los/as futuros/as profesionales todos los elementos evidenciados. Es preciso, por lo tanto, incluir la obligatoriedad de materias de Trabajo Social y salud en las que se delimiten conceptualmente tanto el Trabajo Social sanitario como el sociosanitario, y se incida en su desarrollo profesional. Por otro lado, la creación de programas de postgrado específicos en diferentes universidades favorecería la mayor visibilidad de profesionales e investigadores especializados, lo cual implicaría la mayor presencia de documentos científicos al respecto. Así mismo, sería idóneo promover, en el ámbito académico y profesional de nuestro contexto, una reivindicación de la especialización para ambas tipologías, abogar por un proceder que supere la mera gestión y coordinación de recursos (Gijón y Colom, 2016), y por la transdisciplinariedad.

\section{Referencias bibliográficas}

Álamo, M.T. del y Ruiz, M. (2017). Análisis de la calidad de vida de las personas con discapacidad intelectual: Un estudio comparativo entre gitanos y no gitanos. Cuadernos de Trabajo Social, 30(2), 451-461.

Arza, J. y Carrón, J. (2016). Competencia intercultural en el ámbito de las drogodependencias. Cuadernos de Trabajo Social, 29(1), 109-118.

Brufao, C. (1994). Una aproximación a las enfermedades profesionales del policía. Cuadernos de Trabajo Social, 7, 251-264.

Casal, J.C. (2010). Derechos y participación de las personas con diversidad mental. Cuadernos de Trabajo Social, 23, 301-322.

Castillo, A. y Abad, G. (1991). Las funciones del trabajador social en los equipos de atención primaria del Insalud. Cuadernos de Trabajo Social, 4-5, 129-138.

Colom, D. (2010). El trabajo social sanitario en el marco de la optimización y sostenibilidad del sistema sanitario. Zerbitzuan: Gizarte zerbitzuetarako aldizkaria= Revista de servicios sociales, 47, 109-119.

Coloma, A. (2009). Una aproximación a la intervención del Trabajo Social comunitario en situaciones de catástrofes y desastres. Cuadernos de Trabajo Social, 22, 243-257.

Consejo General del Trabajo Social. (s. f.). La especialización en Ciencias de la Salud del Trabajo Social. Recuperado de: http:/www.cgtrabajosocial.es/app/webroot/files/consejo/files/La\%20especializaci\%C3\%B3n\%20en\%20Ciencias\%20de\%20la\%20Salud\%20del\%20TS\%202013.pdf

Cordero, N. y Palacios, J.E. (2017). Claves éticas para el Trabajo Social, la dependencia, el cuidado y la autonomía. Cuadernos de Trabajo Social, 30(1), 65-75.

Cury, S.P. (2009). Estudio del diagnóstico social en residencias para personas mayores asistidas en la Comunidad de Madrid: diseño de un instrumento de valoración y diagnóstico social. Cuadernos de Trabajo Social, 22, 201-226.

Desviat, M. (2017). «Precariado» y control social: asistencialismo y exclusión en el ámbito de la salud mental. Cuadernos de Trabajo Social, 30(2), 369-376.

Fernández-Riquelme, S. (2015). Teoría de la intervención social. Fundamentos y modelos para el Trabajo Social. Murcia: Diego Marín.

Ferro, S. (2004). Interdisciplinariedad y drogodependencias. Cuadernos de Trabajo Social, 17, 289-304.

Garcés, E.M. (2010). El Trabajo Social en Salud Mental. Cuadernos de Trabajo Social, 23, 333-352.

Gil, D. (2010). Trabajo Social y atención temprana en psicosis, una propuesta de intervención. Cuadernos de Trabajo Social, 23, 403-418.

Gijón, M.T. y Colom, D. (2016). Debates transdisciplinares en torno a la especialización del trabajo social sanitario. En: D. Carbonero, E. Raya, N. Caparrós y C. Gimeno, Respuestas transdisciplinares en una sociedad global: Aportaciones desde el Trabajo Social (pp.1-23). Logroño: Universidad de La Rioja. Recuperado de: https://publicaciones.unirioja.es/catalogo/online/CIFETS_2016/Monografia/pdf/ TC376.pdf

Hernández, R., Fernández, C. y Baptista, P. (2003). Metodología de la investigación. México: McGraw-Hi11 .

Lamas, M. (2014). Cuerpo, sexo y política. Ciudad de México: Océano. 
BOE. (1986, 28/04). Ley 14/1986, de 25 de abril, General de Sanidad. BOE, 102. Recuperado de: http:// noticias.juridicas.com/base datos/Admin/114-1986.html

BOE. (2006, 15/12). Ley 39/2006, de 14 de diciembre, de Promoción de la Autonomía Personal y Atención a las personas en situación de dependencia. BOE, 299. Recuperado en https://www.boe.es/buscar/act. php?id=BOE-A-2006-21990

Martínez, F.M., Sánchez, J. y López, J.L. (2009). El metaanálisis en el ámbito de las Ciencias de la Salud: una metodología imprescindible para la eficiente acumulación del conocimiento. Fisioterapia, 31(3), $107-114$.

Martínez, N. y Ruano, S. (2013). Lo que el síntoma esconde: un caso de abuso sexual. Cuadernos de Trabajo Social, 26(2), 305-314.

Mercado, E. y García, L.M. (2010). Necesidades sociales de las personas con discapacidad en edad escolar y sus familias. Cuadernos de Trabajo Social, 23, 9-24.

Millán, R. (2005). Intervención social grupal. Integrando la perspectiva de género (promoción de relaciones saludables y buen trato). Cuadernos de Trabajo Social, 18, 333-350.

Ministerio de Sanidad, Servicios Sociales e Igualdad. (2014). Plan Nacional de Acción para la Inclusión Social del Reino de España 2013-2016. Madrid: Centro de publicaciones del Ministerio de Sanidad, Servicios Sociales e Igualdad. Recuperado de: https://www.msssi.gob.es/ssi/familiasInfancia/inclusionSocial/docs/PlanNacionalAccionInclusionSocial_2013_2016.pdf

Núñez, G. (1999). Trabajo Social de casos/familia en centros de salud de atención primaria (INSALUD). Cuadernos de Trabajo Social, 12, 185-198.

Núñez, G. y Cerón, A. (2001). Proyecto de trabajo grupal con mujeres cuidadoras principales informales de familiares mayores dependientes. Cuadernos de Trabajo Social, 14, 353-362.

OMS.(s.f.). Temas de salud. Factores de riesgo. Recuperado de: http://www.who.int/topics/risk factors/es/

Pérez, M.T. (2012). Salud: marco teórico y conceptual. En: A, Hidalgo y L.P. Martín, Salud Pública, Dependencia y Trabajo Social (pp. 21-50). Madrid: Ediciones Académicas.

Pérez López, R.B. (1990). Trabajo Social y salud: la teoría hecha práctica para una transformación social. Cuadernos de Trabajo Social, 3, 143-157.

Puente, A. (2001). Psicobiología de la memoria: Evolución de las alteraciones de memoria en las personas mayores y los enfermos tipo Alzheimer. Cuadernos de Trabajo Social, 14, 185-220.

Robles, P. y Lara, F. (2009). Evaluación del Servicio de Ayuda a Domicilio en Alcorcón: diagnóstico para la mejora de la calidad del servicio. Cuadernos de Trabajo Social, 22, 259-276.

Rodríguez, C. (2013). Las familias y los cuidados a las personas mayores dependientes: entre la reciprocidad y la ambivalencia. Cuadernos de Trabajo Social, 26(2), 349-358.

Rodríguez-Otero, L.M. (2016). El arraigo de las violencias. La violencia intragénero. Ciudad de México: Grañén Porrúa.

Sánchez, M., Sotomayor, R., Aquino, R., Rodríguez, R.P., Gil, J.M. y Gualda, R. (2011). El trabajador social como profesional del Sistema Sanitario. Sevilla: Eduforma.

Sanz, A. (2009). ¿Cómo piensan y viven los adolescentes su sexualidad?: resumen de una memoria de investigación. Cuadernos de Trabajo Social, 22, 277-296.

Servillo, A. (1999). Una mirada epistemológica sobre la salud. Recuperado de: https://psico.edu.uy/sites/ default/files/cursos/taller_2dociclo_ficha5_pdf_22967.pdf

Ureña, A. (2010). La salud mental del Trabajo Social en Cataluña. Cuadernos de Trabajo Social, 23, $353-$ 360.

Vázquez, O. (2004). Informe de la Comisión de Evaluación del diseño del Título de Grado en Trabajo Social. Madrid: Agencia Nacional de Evaluación de la Calidad y Acreditación. 
Figura 3: Evolución legislativa en España en materia de Política Social.

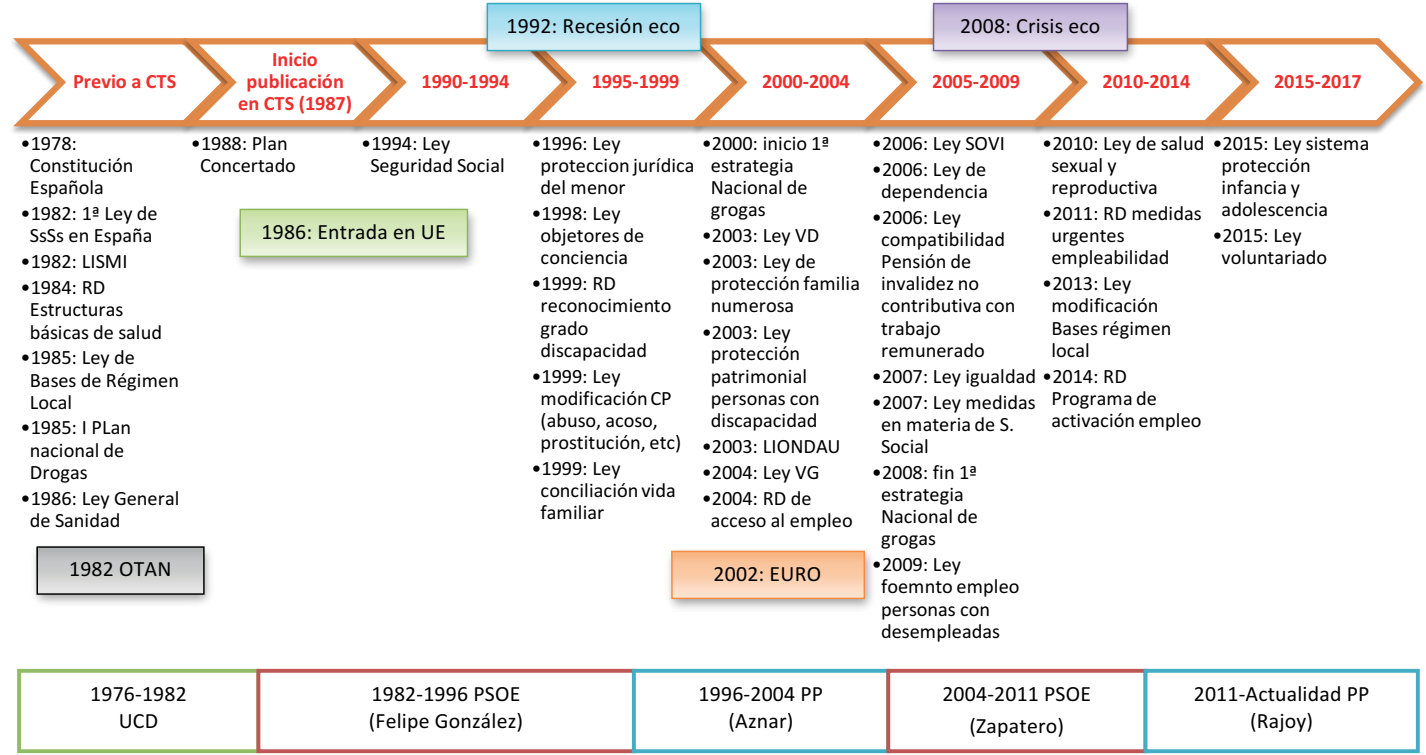

